\title{
COMPARISON OF POULTICE-TYPE AND TAPE-TYPE PATCHES CONTAINING KETOPROFEN ON HUMAN SKIN IRRITATION
}

\author{
Naohisa KAWAMURA, Norihiro SHINKAI, Hitoshi YAMAUCHI and Satoshi TAKAYAMA \\ Research Department, Saitama Daiichi Pharmaceutical Co., Ltd., \\ 8-1 Minamisakae-cho Kasukabe, Saitama 344-0057, Japan
}

(Received June 27, 2003; Accepted September 9, 2003)

\begin{abstract}
Although the patch test for visual skin observation is widely used clinically, it does not allow the mechanisms of side effects to be assessed. In this study, we examined poultice-type KP801 and tape-type KP-T patches containing ketoprofen. The parameters to measure side effects on skin were peeling intensity, amount of stripped stratum corneum, skin moisture and redness of skin color under various mechanical conditions.

Since the amount of stripped stratum corneum with the tape-type KP-T patch was higher than with the poultice-type KP801 patch, the bio-adhesive strength of the latter was concluded to be lower. A clear relationship exists between the amount of stripped stratum corneum and skin moisture after tape-type patch removal, but this was not found with the poultice-type patch because of its hydration effects. Peeling intensity, one parameter to predict pain at the time of patch removal, was higher with the KP-T. As for mechanical conditions, when the patch is removed, it is important to remove it as slowly as possible and horizontally, and to avoid any rise in skin temperature. Finally, when a patch is applied to a region with little skin moisture, the amount of stripped stratum corneum may increase accordingly.
\end{abstract}

KEY WORDS: Skin irritation, Healthy human volunteers, Poultice-type patch, Tape-type patch, Ketoprofen

\section{INTRODUCTION}

Transdermal administration of drugs has many advantages when compared with oral or injection routes, such as the avoidance of the first pass effect in the liver and side effects on the gastrointestinal tract, a prolonged steady-state blood concentration of the drug for a long period of time and fast discontinuation on removal (Chien, 1992; Kinoshita et al., 1993). The skin has a large surface area of about $1.8 \mathrm{~m}^{2}$ and consists of the stratum corneum, epidermis, dermis and attached elements, such as the hair follicle, sebaceous gland and sweat gland (Schaefer and Redelmeier, 1996a). The stratum corneum of the outermost layer plays an important role to prevent not only water loss through the skin, but also penetration of foreign substances from the outside (Schaefer and Redelmeier, 1996b).

Skin irritation caused by transdermal medication is due to chemical, biological and physical mechanisms. The chemical, biological and physical mecha- nisms involve direct toxicity of drug molecules, inhibition of respiration and excretion through the skin, and peeling intensity or stripping of the skin surface, respectively (Sugii et al., 1989; Kinoshita et al., 1993; Tomita and Tagami, 1993; Inoue et al., 1994; Iizuka et al., 1998). The biological mechanisms are more complicated and include direct or indirect bio-reactions as in allergies. In these irritations, as an evaluation method of the physical skin irritation, although in vivo evaluation methods for human skin irritation are limited, skin color observation using a spectro-photometer (Andersen et al., 1991; Kinoshita et al., 1993; Takiwaki and Serup, 1994; Shirai et al., 1994) and electrical conductivity (Tagami et al., 1980; Tagami, 1981, 1982; Obata and Tagami, 1989; Kinoshita et al., 1993), are both important methods which can be applied to test the safety of transdermal formulations.

There are basically two types of patches, one of the poultice-type which consists of water-soluble polymer and contains a lot of water and the other of the 
tape-type consisting of hydrophobic polymers without water. Ketoprofen is a conventional non-steroidal antiinflammatory drug (NSAID) that exhibits non-selective cyclooxygenase inhibition (Warner et al., 1999; Kay-Mugford et al., 2000) and skin permeability of ketoprofen from water or acetone solution was higher than those of other NSAIDs (Yano et al., 1986; Cordero et al., 1997). These characteristics make ketoprofen highly suitable for topical application using either type of patch. Although both types of patches containing ketoprofen are already marketed and prescribed for a lot of patients, there has been no report regarding the difference of safety between two types of patches as compared directly and occurrence of physical skin irritation according to the method of patch removal.

In the present study, we investigated the physical skin irritation comparison between the poultice-type patch and the tape-type patch containing ketoprofen in various mechanical conditions.

\section{MATERIALS AND METHODS}

\section{Test patches}

The KP801 poultice-type patch $\left(\right.$ MILTAX $\left.^{\circledR}\right)$, which contains $0.3 \%$ ketoprofen, and $56 \%$ water with some water-soluble polymers like sodium polyacrylate, sodium carmellose and gelatin, was obtained from Saitama Daiichi Pharmaceutical Co., Ltd (Saitama, Japan). The KP-T tape-type patch (MOHRUS ${ }^{\circledR}$ TAPE, Hisamitsu Pharmacetical Co., Inc., Japan), which contains $2 \%$ ketoprofen in hydrophobic adhesive like styrene/isoprene/styrene copolymer without water, was purchased from Asahi Pharmacy (Tokyo, Japan). Both patches are widely used clinically.

\section{Study Design}

Three examinations evaluated the influences of the difference in the application regions, the removal speed, the removal angle and the temperature at the application region.

A comparison of temperature at the application region was studied between room temperature, the skin surface temperature: $33^{\circ} \mathrm{C}$, and warm condition, the skin surface temperature: $38^{\circ} \mathrm{C}$, with the portable body warmer; CAIRO (Nittetsu, Japan). The temperature was measured with a DTM900 digital thermometer (MT, Japan) before the patch application and just before the patch removal.

Eleven healthy male volunteers between $25-45$ years old gave written consent to participate in this study, which was approved by the Institutional Review
Board of Saitama Daiichi Pharmaceutical Co., Ltd.

\section{Application of the patch}

Before the examination, the amount of skin moisture in the application region was measured using SKICON 200 (IBS Co., Ltd., Hamamatsu, Japan) under controlled conditions of $23^{\circ} \mathrm{C}$ and $65 \%$ relative humidity. After light wiping of the application region with a wet towel $30 \mathrm{~min}$ before the patch application, the volunteers stayed in a controlled room for $15 \mathrm{~min}$ or more. Then, $2.0 \mathrm{~cm} \times 3.5 \mathrm{~cm}$ patches were applied to the test site and removed after $10 \mathrm{hr}$. According to the JIS (Japanese Standards Association, 1991) test method, the patch was removed at rates of 300 or $1800 \mathrm{~mm} / \mathrm{min}$ using a Sun Rheo Meter CR-200D (Sun Scientific Co., Ltd., Japan) or by hand in the controlled room. The peeling intensity with the patch, amount of stripped stratum corneum, redness of skin color and amount of skin moisture were measured as detailed below.

\section{Measurement of peeling intensity}

The maximum and minimum values for the removal intensity of the patch were read on a recorder using the Sun Rheo Meter CR-200D, and the average value was represented as the peeling intensity $(\mathrm{g} / 2.0$ $\mathrm{cm})$ with the patch.

\section{Measurement of amounts of stripped stratum cor- neum}

The adhesion side of KP801 removed was stuck to the adhesion side of pack tape (No.451, Teraoka, Japan) for transfer of the stripped stratum corneum adhering to the patch. This operation was repeated five times and five samples per patch were obtained. The pack tape was then immersed for $24 \mathrm{hr}$ in dye solution (brilliant blue: water $=1: 99$ ). In the case of KP-T, the removed patch was directly immersed in the dyeing solution. After washing off excess dye with water, the sample was punched in a diameter of $18 \mathrm{~mm}$. The dyeing matter of the sample was extracted with the help of ultrasonic waves in extraction solution (benzalkonium chloride: water $=1: 99$ ) and the absorbance (wavelength: $630 \mathrm{~nm}$ ) of each sample was measured with a Viento Multi-Spectrophotometer (Dainippon Pharm., Japan).

\section{Measurement of redness of skin color and amount of skin moisture}

Thirty minutes and $24 \mathrm{hr}$ after the patch removal, the redness of skin color in the application region was measured using an SP-64 (X-Rite, Inc., USA). Within 
Skin irritation due to patches.

$1 \mathrm{hr}$ after the patch removal, the amount of skin moisture (conductance; Mic. S) was measured using a SKICON 200.

\section{Statistical analysis}

The results are expressed as mean \pm S.E. values. Statistical significance was determined by multiple comparison with the Tukey test (Tukey, 1953) and the $t$ test for comparison of two samples. The criterion for significance was $\mathrm{p}<0.05$ with all statistical evaluations.

\section{RESULTS}

Influence of various application regions on amounts of stripped stratum corneum, skin moisture and redness of skin color for KP801

At the speed of $300 \mathrm{~mm} / \mathrm{min}$, when KP801 was removed horizontally $\left(180^{\circ}\right)$ after $10 \mathrm{hr}$ of application on the forearm, the shoulder, the elbow and the knee, the amounts of stripped stratum corneum, skin moisture and redness of skin color were as summarized in
Fig. 1.

The amount of stripped stratum corneum was in the order of knee $>$ forearm $>$ elbow $>$ shoulder. The ratios of the amount of skin moisture (after/before application of KP801) are shown in Fig. 2. In all application regions, the amount of skin moisture was markedly increased 4 to 6 times after the application of KP801 for $10 \mathrm{hr}$. No significant difference in redness of skin color was observed among the application sites.

Influence of various mechanical conditions on peeling intensity, amounts of stripped stratum corneum, skin moisture and redness of skin color for KP801 and KP-T

1. Comparison between KP801 and KP-T on peeling intensity, amounts of stripped stratum corneum, skin moisture and redness of skin color in usual use condition (removal speed: $300 \mathrm{~mm} /$ min, removal angle: $180^{\circ}$, application site temperature: $33^{\circ} \mathrm{C}$ )

The peeling intensity, amount of stripped stratum

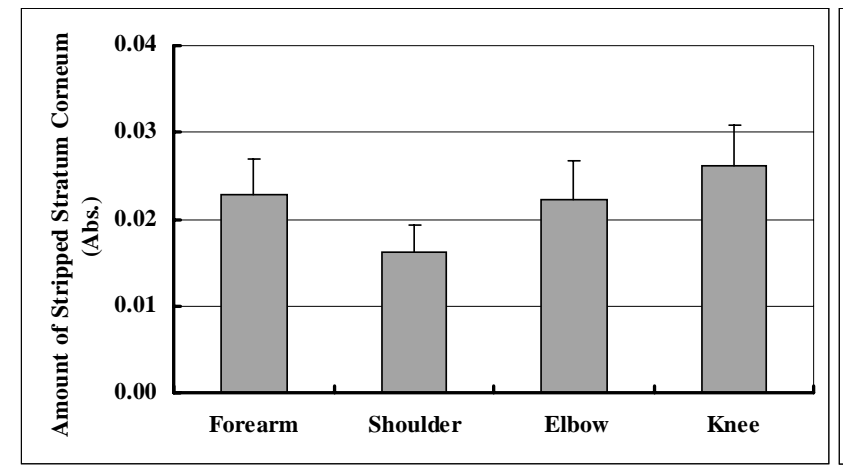

1-a)

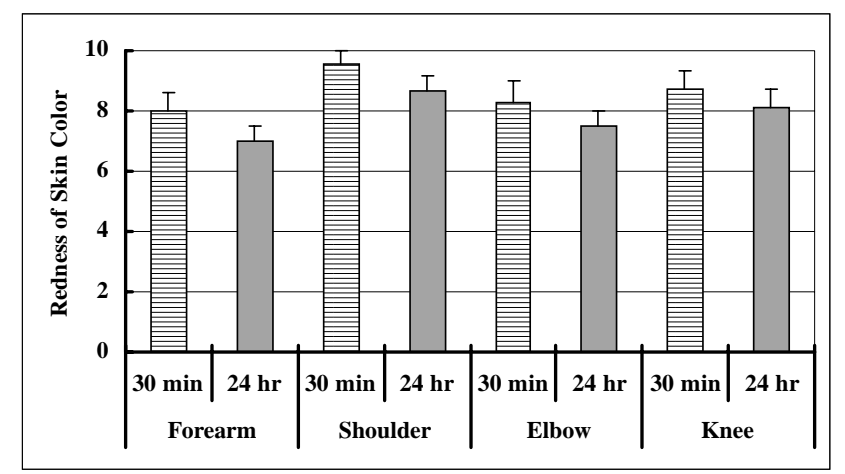

1-c)

Fig. 1. Comparison of amount of stripped stratum corneum (1-a), amount of skin moisture (1-b) and redness of skin color (1-c) with different application regions (forearm, shoulder, elbow and knee) for KP801 under standard conditions.

Data are mean \pm S.E. values for eleven volunteers. Each poultice was applied for $10 \mathrm{hr} . * * \mathrm{p}<0.01 \mathrm{vs.} \mathrm{Forearm} \mathrm{(Before} \mathrm{application).}$

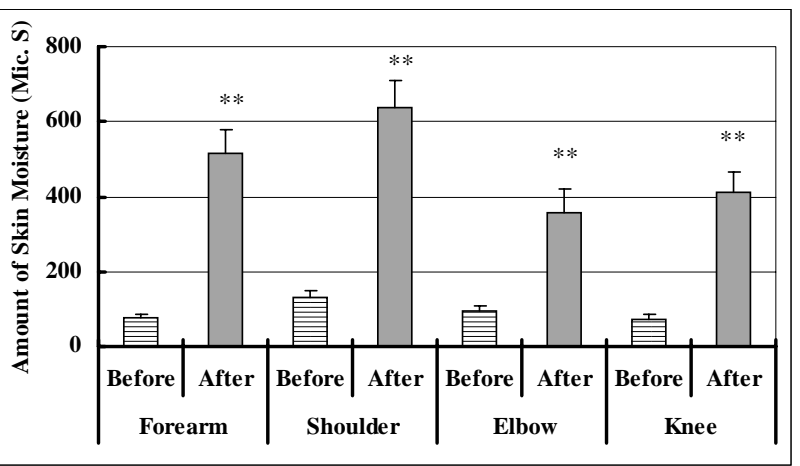

1-b) 
N. KAWAMURA et al.

corneum, amount of skin moisture and redness of skin color were compared between KP801 and KP-T, and the results are shown in Fig. 3.
Although no difference in redness was observed, the peeling intensity and the amount of stripped stratum corneum with KP-T were higher than with KP801.

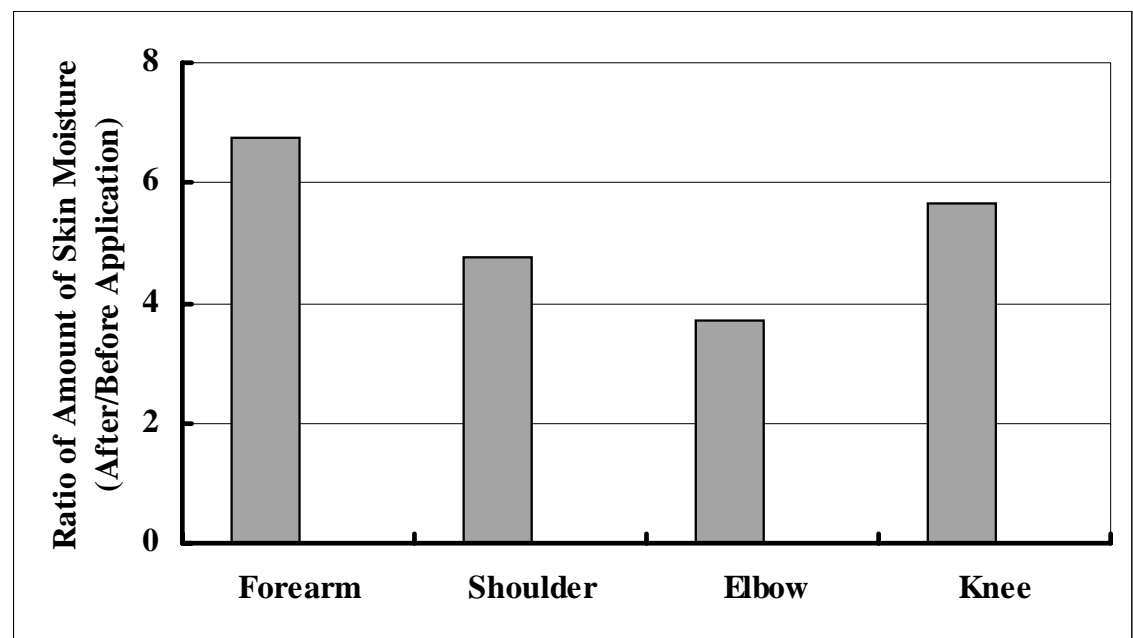

Fig. 2. Ratios of before and after application of KP801 for the amount of skin moisture with the different application regions.

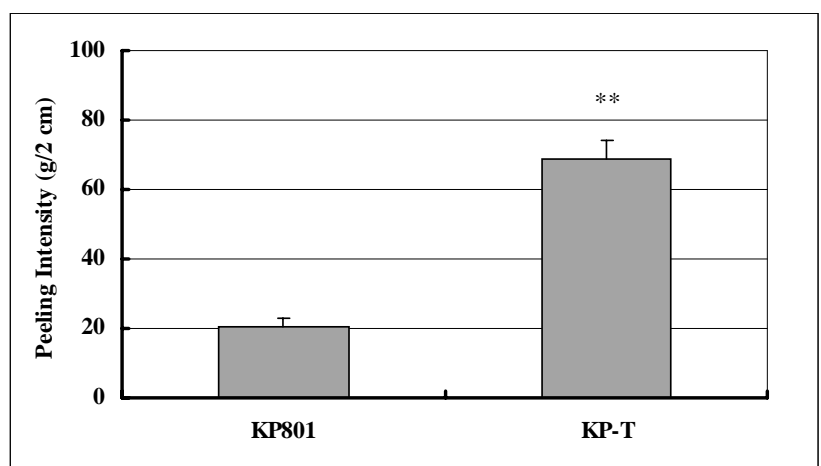

3-a)

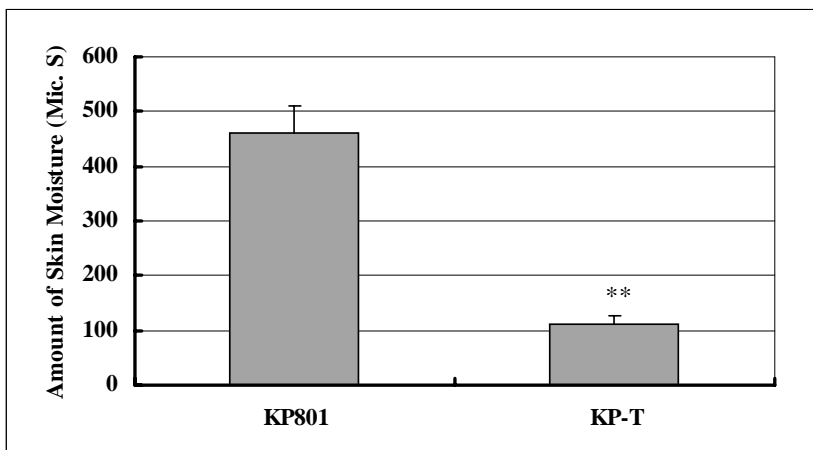

3-c)

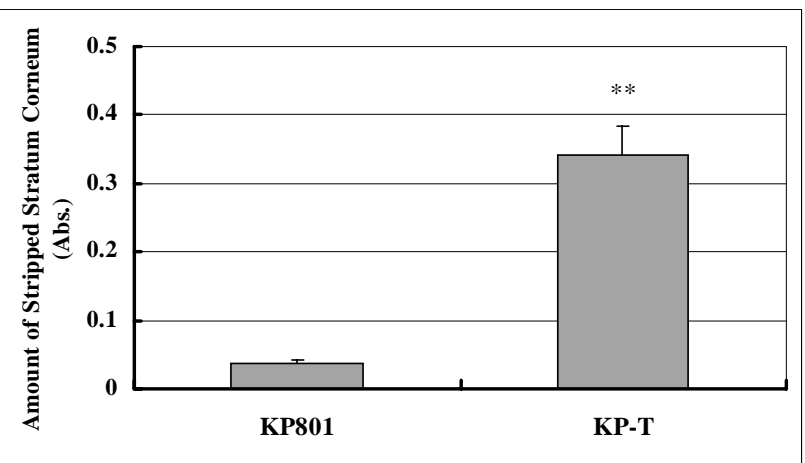

3-b)

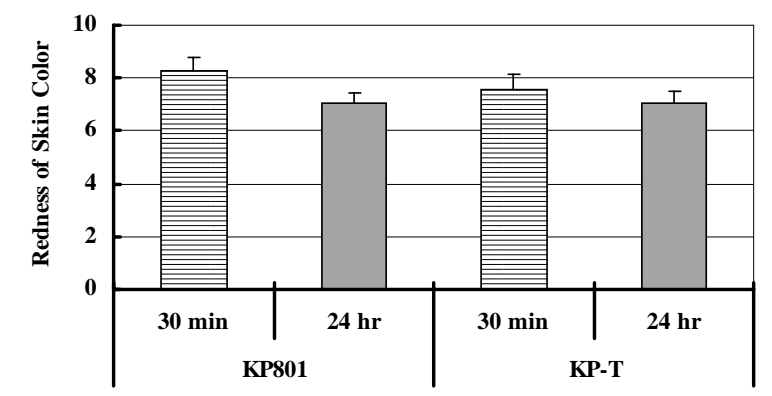

3-d)

Fig. 3. Comparison of peeling intensity (3-a), amount of stripped stratum corneum (3-b), amount of skin moisture (3-c) and redness of skin color (3-d) with KP801 and KP-T under standard conditions.

Data are mean \pm S.E. values for eleven volunteers. Each patch was applied on the forearm for $10 \mathrm{hr} .{ }^{* *} \mathrm{p}<0.01 \mathrm{vs}$. KP801. 
Skin irritation due to patches.

The amount of skin moisture was higher after KP801 treatment.

\section{Influence of removal speed ( $300 \mathrm{vs} 1800 \mathrm{~mm} / \mathrm{min}$ ) on amounts of stripped stratum corneum, skin moisture and redness of skin color for KP801 and KP-T}

At the speed of 300 or $1800 \mathrm{~mm} / \mathrm{min}, \mathrm{KP} 801$ and KP-T were removed horizontally $\left(180^{\circ}\right)$ after $10 \mathrm{hr}$ of application to the forearm. The results for amount of stripped stratum corneum, skin moisture and redness of skin color are shown in Fig. 4.

While redness was not affected, the amounts of stripped stratum corneum and skin moisture were higher when the patches were removed at the quick speed of $1800 \mathrm{~mm} / \mathrm{min}$, and the amount of stripped stratum corneum was higher and amount of skin moisture was lower after the treatment of KP-T than with KP801.
3. Influence of removal angle $\left(180 \mathrm{vs} 90^{\circ}\right)$ on peeling intensity, amounts of stripped stratum corneum, skin moisture and redness of skin color for KP801 and KP-T

At the speed of $300 \mathrm{~mm} / \mathrm{min}, \mathrm{KP} 801$ and KP-T were removed at angles of 180 or $90^{\circ}$ after $10 \mathrm{hr}$ of application. The results for peeling intensity, amount of stripped stratum, amount of skin moisture and redness of skin color are shown in Fig. 5.

The peeling intensity was higher with $90^{\circ}$ than the $180^{\circ}$ angle. On the other hand, the removal angle did not have a notable influence on the amount of stripped stratum corneum, skin moisture and redness of skin color with either patch. Although the amount of skin moisture was lower with the two removal angles with KP-T, the peeling intensity and amounts of stripped stratum corneum were higher than those for KP801. No obvious difference in redness of skin color was evident between the two patches.

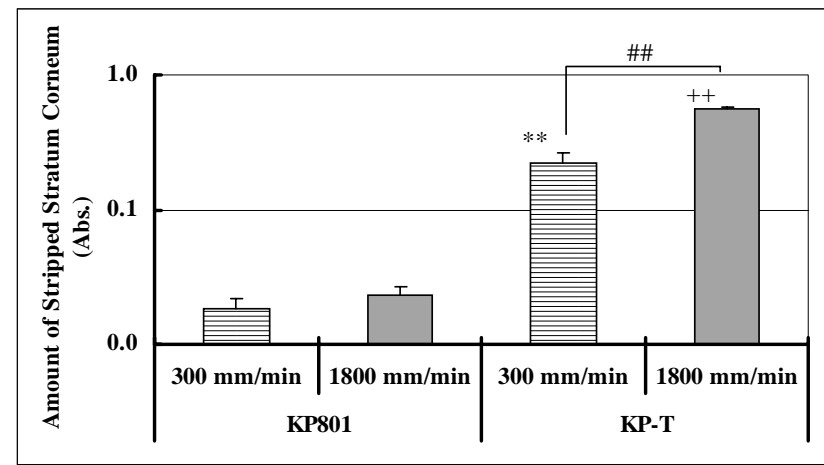

4-a)

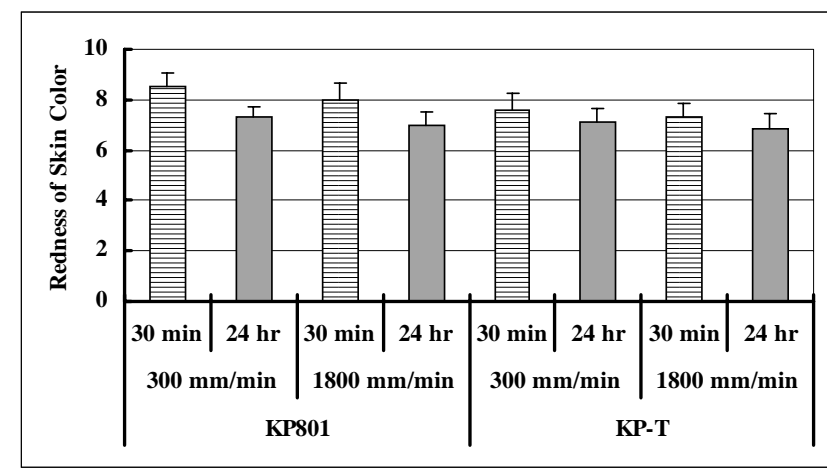

4-c)

Fig. 4. Effects of removal speed on amount of stripped stratum corneum (4-a), amount of skin moisture (4-b) and redness of skin color (4-c) with KP801 and KP-T.

Data are mean \pm S.E. values for eleven volunteers. Each patch was applied on the forearm for $10 \mathrm{hr}$. $* * \mathrm{p}<0.01 \mathrm{vs.} \mathrm{KP801}$ at 300 $\mathrm{mm} / \mathrm{min} .++\mathrm{p}<0.01$ vs. KP801 at $1800 \mathrm{~mm} / \mathrm{min}$. \#\#p<0.01 vs. KP-T at $300 \mathrm{~mm} / \mathrm{min}$.

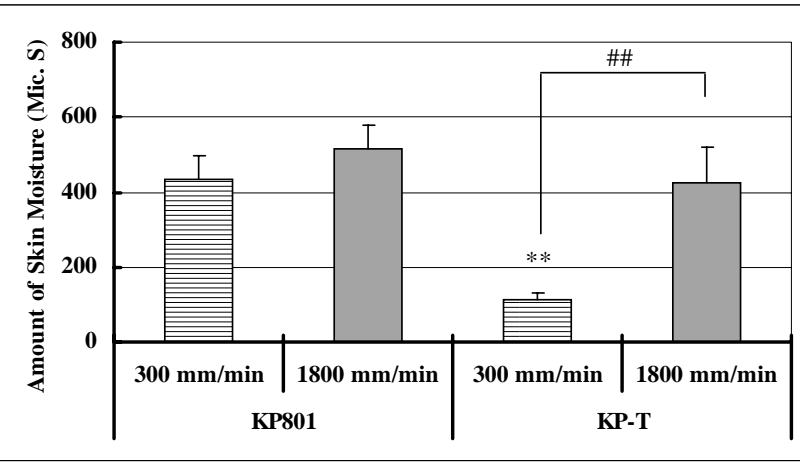

4-b) 
4. Influence of the application site temperature (33 vs $38^{\circ}$ ) on peeling intensity, amounts of stripped stratum corneum, skin moisture and redness of skin color for KP801 and KP-T

At a speed of $300 \mathrm{~mm} / \mathrm{min}$ and an angle of $180^{\circ}$, a comparison between KP801 and KP-T at $33^{\circ} \mathrm{C}$ and $38^{\circ} \mathrm{C}$ is revealed in Fig. 6.

Peeling intensity with KP801 tends to be higher under a warm condition. Although the peeling intensity of KP-T was higher than that of KP801, it was slightly lower at $38^{\circ} \mathrm{C}$. The amount of stripped stratum corneum was higher with KP-T than with KP801, while the amount of skin moisture was higher with KP801 under the warm condition. No difference in the redness of skin color was noted with the two patches at different temperatures.

\section{DISCUSSION}

There are several formulations for topical appli- cation of drugs, such as patches, ointments, lotions and sprays. Although each possesses advantages and disadvantages, patches are widely used because of their practical utility, satisfaction of QOL (quality of life) for the patients and their superior characteristics for efficacy. However, severe side effects of patches may be shown on the skin, such as contact dermatitis, allergy and phototoxicity. Since a species difference has been noted among animals, it is very difficult to predict side effects on human skin from animal toxicity studies (Rosati et al., 1992; Masuda, 1995). At the time of the patch removal, when the amount of stripped stratum corneum is large, skin irritation is reinforced (Kinoshita et al., 1993; Tomita and Tagami, 1993; Inoue et al., 1994; Iizuka et al., 1998).

In the present study, the amount of stripped stratum corneum with KP-T was higher than with KP801 under all conditions, so that we can conclude that the bio-adhesive strength of this tape-type patch is stronger than that of KP801. The amount of stripped stratum

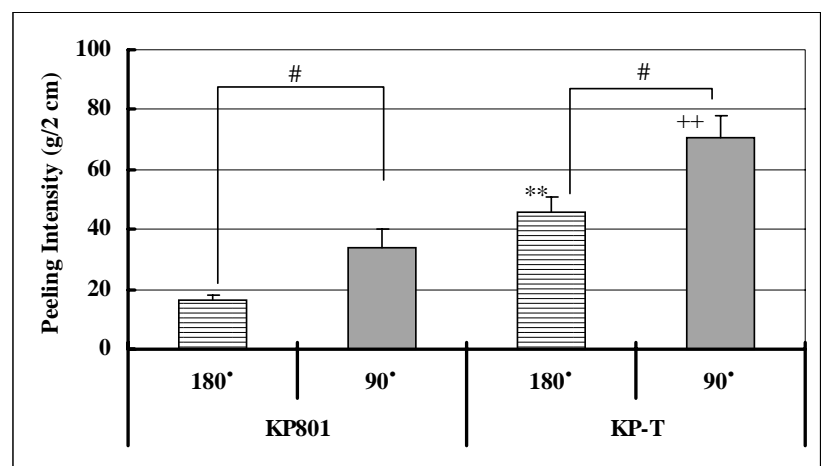

5-a)

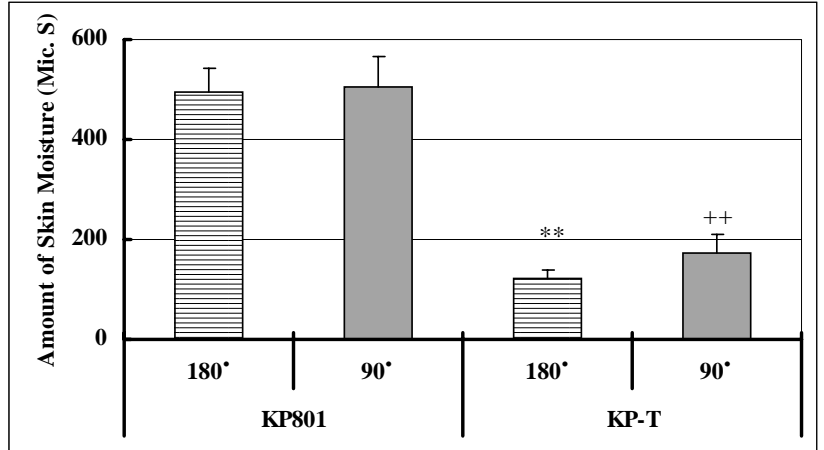

5-c)

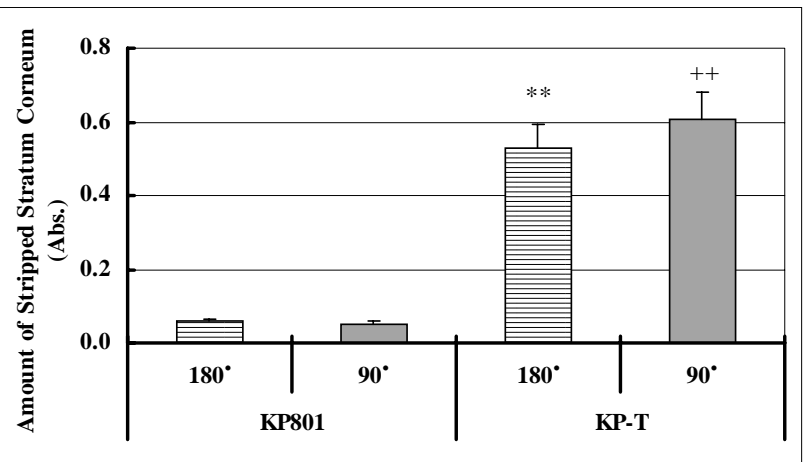

5-b)

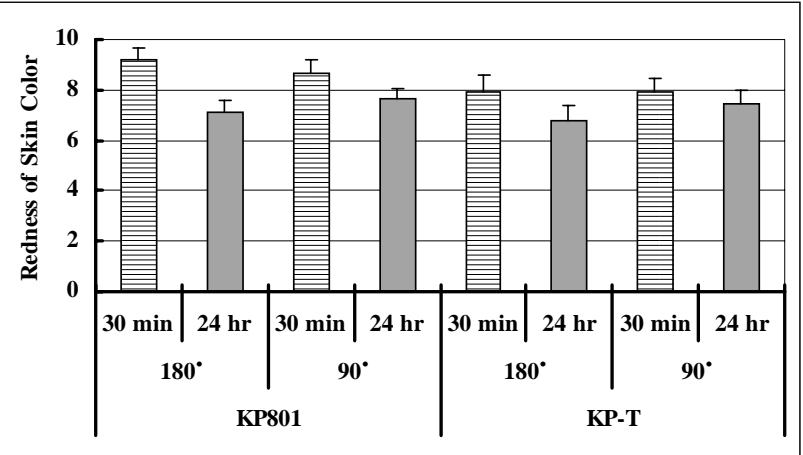

5-d)

Fig. 5. Effects of removal angle on peeling intensity (5-a), amount of stripped stratum corneum (5-b), amount of skin moisture (5-c) and redness of skin color (5-d) with KP801 and KP-T.

Data are mean \pm S.E. values for eleven volunteers. Each patch was applied on the forearm for $10 \mathrm{hr} .{ }^{* *} \mathrm{p}<0.01 \mathrm{vs.} \mathrm{KP801}$ at 180 degrees, $++p<0.01$ vs. KP801 at 90 degrees, $\# p<0.05$ vs. each patch at 180 degrees. 
Skin irritation due to patches.

corneum increased when the patch was removed at the quick speed of $1800 \mathrm{~mm} / \mathrm{min}$, so that removal should be as slow as possible, as recommended on patch labels. It appears that perpendicular as opposed to horizontal removal is not of major importance.

For the purpose of reinforcing the bio-adhesion, KP801 is blended with gelatin, this latter demonstrating reversible fluctuation between a sol and a gel at approximately $35^{\circ} \mathrm{C}$ (Keiei Kaihatsu Center Syuppanbu, 1981). This reaction is based on hydrogen bonds between NH and CO residues (CMC, 1968), the polar groups reinforcing adhesive strength according to Van der Waals' or hydrogen bond forces (Nihon Nenchakuzai Tape Kogyokai, 1995). It is known that this fluidity with change of temperature increases wetting of the skin, so that adhesion is reinforced (Nihon Nenchakuzai Tape Kogyokai, 1995). There are reports suggesting higher irritation in the summer season, and thus the comparison between $33^{\circ} \mathrm{C}$, the usual skin temperature, and $38^{\circ} \mathrm{C}$ was conducted. The amount of stripped stratum corneum of KP801 under the warm condition was higher, because the gelatin became a sol and the patch was wet. Since KP-T consists of styrene/ isoprene/styrene copolymer, whose elasticity depends on temperature, the amount of stripped stratum corneum of $\mathrm{KP}-\mathrm{T}$ at $38^{\circ} \mathrm{C}$ was also increased.

As shown in Fig. 7, a negative high correlation $\left(r^{2}=0.937\right)$ was observed between the amount of skin moisture before the patch application and the amount of stripped stratum corneum. In general, transdermal absorption of drugs shows marked variation with the application region (Wester and Maibach, 1995), depending on the thickness of the stratum corneum (Chien, 1992). Dry skin is weak morphologically, because the arrangement of corneocytes is irregular (Kashibuchi and Muramatsu, 1989). Therefore, when the patch is applied to a region with low skin moisture, the stratum corneum may be stripped by patch removal and this would be expected to cause skin irritation.

The amount of skin moisture is affected by the

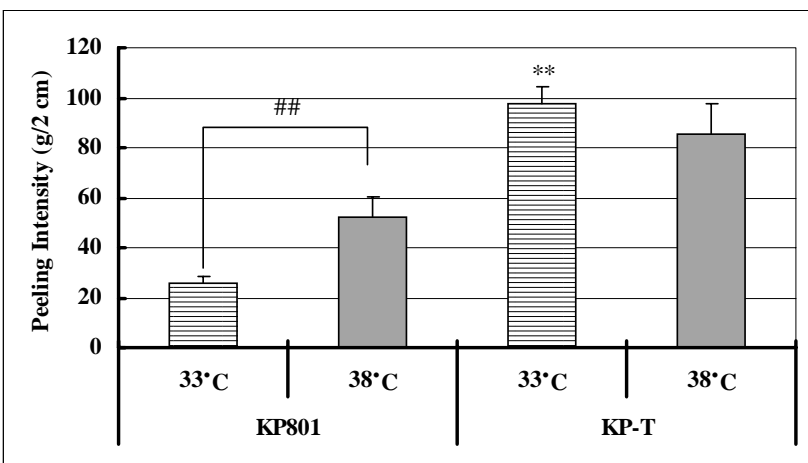

6-a)

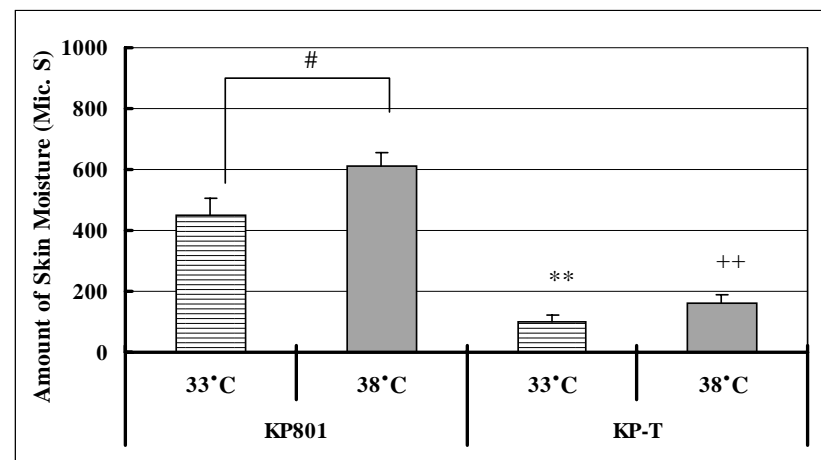

6-c)

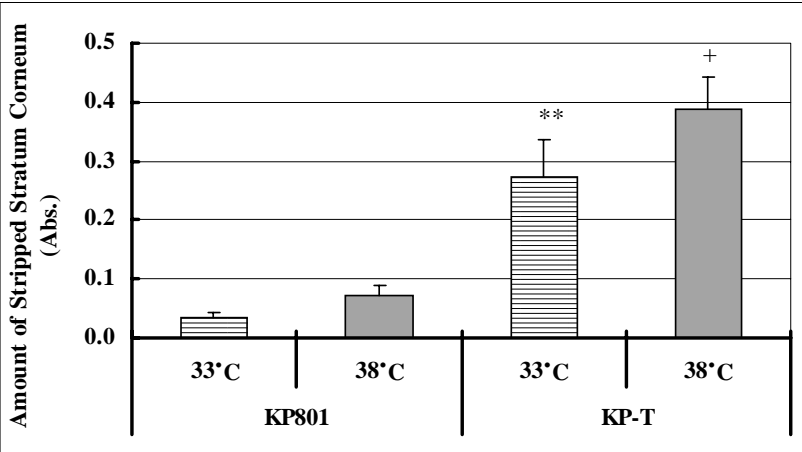

6-b)

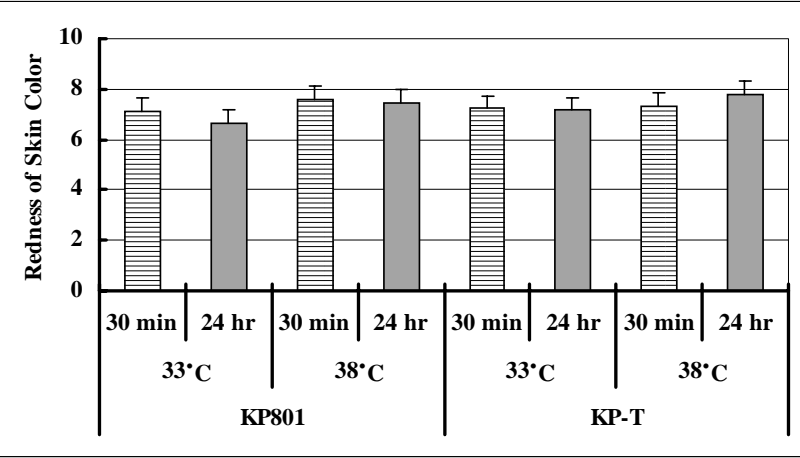

6-d)

Fig. 6. Effects of temperature of the application region on peeling intensity (6-a), amount of stripped stratum corneum (6-b), amount of skin moisture (6-c) and redness of skin color (6-d) of KP801 and KP-T.

Data are mean \pm S.E. values for eleven volunteers. Each patch was applied on the forearm for $10 \mathrm{hr} . * * \mathrm{p}<0.01 \mathrm{vs.} \mathrm{KP801} \mathrm{under}$ room temperature, $+p<0.05 ;++p<0.01$ vs. KP801 under CAIRO, \#p<0.05; \#\#p<0.01 vs. each patch. 
atmospheric water (Yoshikuni et al., 1983), and the occlusive conditions and hydration due to the patch. However, in this study, since we measured the amount of skin moisture under controlled conditions of $23^{\circ} \mathrm{C}$ and $65 \%$ relative humidity, the effect of the atmospheric water can be considered low. Moreover, the effect of occlusion from atmosphere with patch application was rapidly reversible (Schaefer and Redelmeier, 1996c). Our in-house data (Kawakami et $a l ., 2000)$ show that the hydration effect of water in the patch, on the other hand, especially with the poulticetype patch, is sustained for at least one hour. Therefore, in this study, the effect of hydration by water in the patch was more important as a determinant of skin moisture than atmospheric water and occlusion by the patch.

Measurement of trans-epidermal water loss from skin (Flannigan et al., 1985; Schaefer and Redelmeier, 1996b; Zhai et al., 1998; Fluhr et al., 2001) or electrical conductivity (Tagami et al., 1980; Tagami, 1981, 1982; Obata and Tagami, 1989; Kinoshita et al., 1993) is generally used to evaluate skin moisture. We used electrical conductivity in this study, and as shown in Fig. 2, in all application regions, the amounts of skin

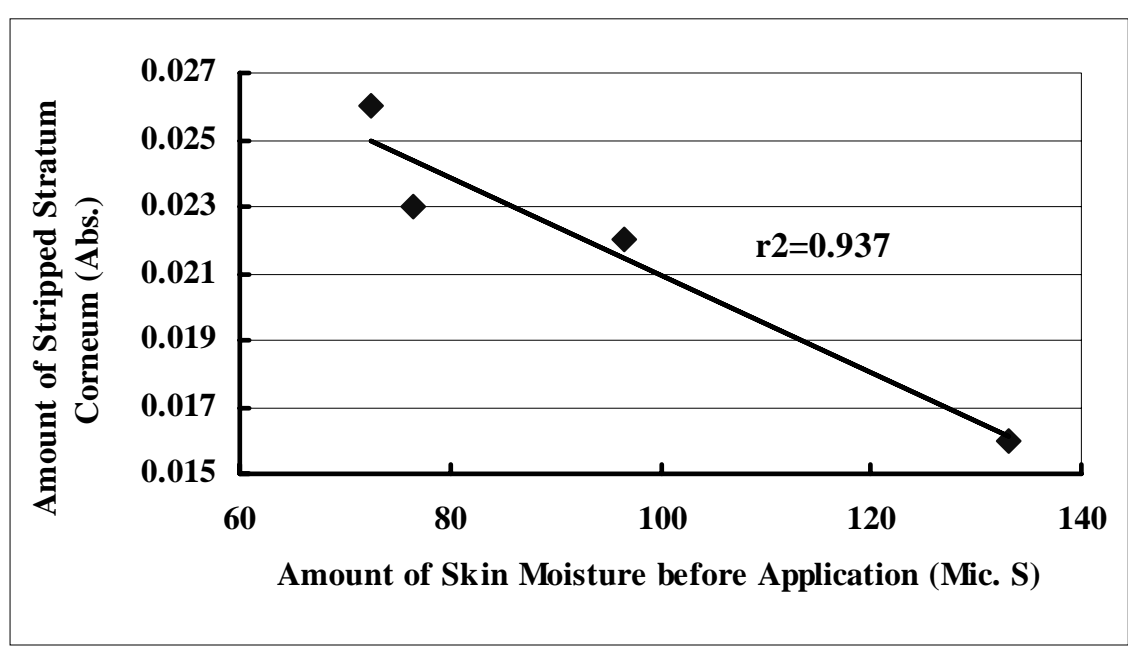

Fig. 7. Relation between amount of skin moisture before application and amount of stripped stratum corneum with KP801.

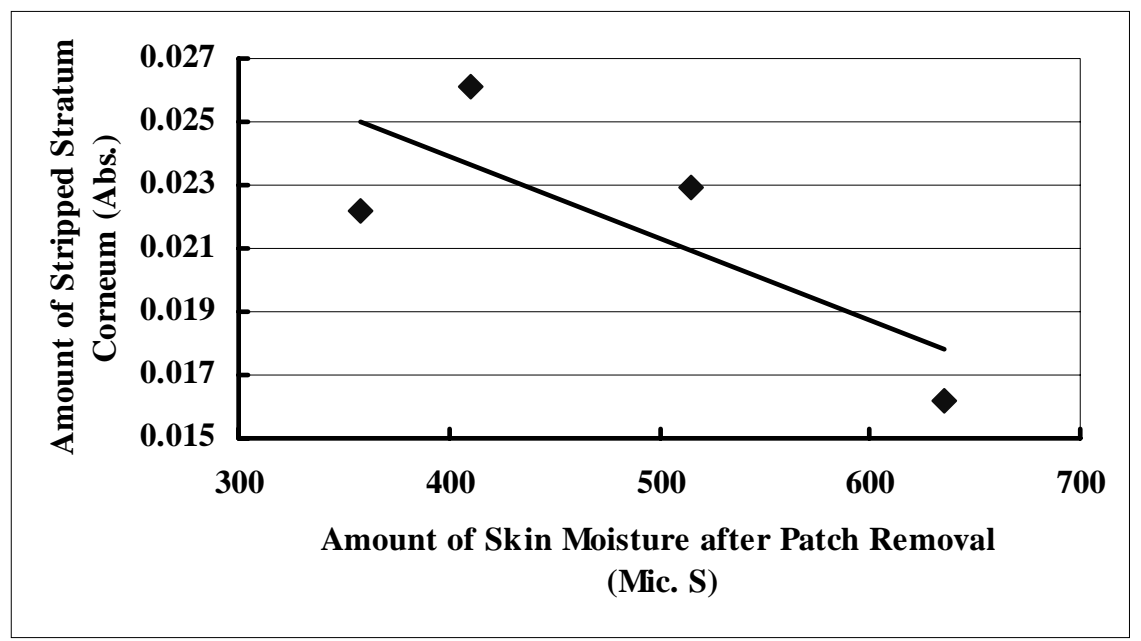

Fig. 8. Relation between amount of skin moisture after patch removal and amount of stripped stratum corneum with KP801. 
Skin irritation due to patches.

moisture after application of KP801 became elevated about 4-6 times. Moreover, as shown in Fig. 8, a negative correlation was observed between the amount of skin moisture after the patch removal and the amount of stripped stratum corneum of KP801 in the different regions. A poultice-type patch with high water content like KP801 may raise the amount of skin moisture and contribute to increase transdermal absorption of a drug and protect the skin (Wester and Maibach, 1999). The fact that skin moisture with KP-T was much lower than with KP801 after the patch removal is in line with the $0 \%$ water content of the tape-type patch.

A good correlation $\left(r^{2}=0.834\right)$ was observed between the water content in the patch and the amount of skin moisture after the patch removal in our inhouse data, as shown in Fig. 9 (Kawamura et al., 2003). Although the amount of skin moisture plays an important role in maintaining skin structure and function (Warner et al., 1988; Kashibuchi and Muramatsu, 1989; Von Zglinicki et al., 1993; Schaefer and Redelmeier, 1996b), both poultice-type and tape-type patches are thought to be safe.

In general, when the amount of stripped stratum corneum increases, the amount of skin moisture also increases (Schaefer and Redelmeier, 1996b). Although there was no clear tendency with KP801 because of its hydration effects, a positive relation between the amount of skin moisture after the patch removal and the amount of stripped stratum corneum was observed with KP-T under all conditions.

The skin penetration of a drug is generally enhanced by occlusive application. Hydration of cor- neocytes causes an increase in the amount of moisture in the intercellular gaps (Schaefer and Redelmeier, 1996d; Bucks and Maibach, 1999) and the structural intensity of the stratum corneum becomes weaker. When the patches were applied at a warm temperature, the amount of skin moisture was elevated, and this might explain the increase in peeling of stratum corneum on patch removal.

When the bio-adhesive strength (peeling intensity (P)) of a patch is strong, removal causes pain (Tomita and Tagami, 1993). The relation to the removal angle $(\mathrm{w})$ is expressed by the following equation (Kaelble, 1959).

\section{$\mathrm{P}=\mathrm{K} /(1-\cos \mathrm{w}) \mathrm{K}$ : constant}

When patches are removed perpendicularly, the distribution area of the stress, which is inflicted on the skin, is larger than that with horizontal removal (Satoh, 1993; Urahama and Tanaka, 1996; Satoh, 1998). Moreover, the location of algesthesia receptors is free nerve endings, which exist in the epidermis (Yokota, 1981; Satoh and Hotta, 1996). Thus, when the patch is removed perpendicularly, it is thought that a lot of algesthesia receptors of the patch application area are stimulated in comparison with horizontal removal. So, when the removal angle is small, the peeling intensity and pain are increased. In this study, the peeling intensity with KP-T was higher than with KP801 under all conditions, reflecting the difference in adhesive and water content in the patches. Moreover, when the patch was removed perpendicularly, the peeling intensity was higher than with horizontal removal, although this did not correlate directly with the amount of stripped

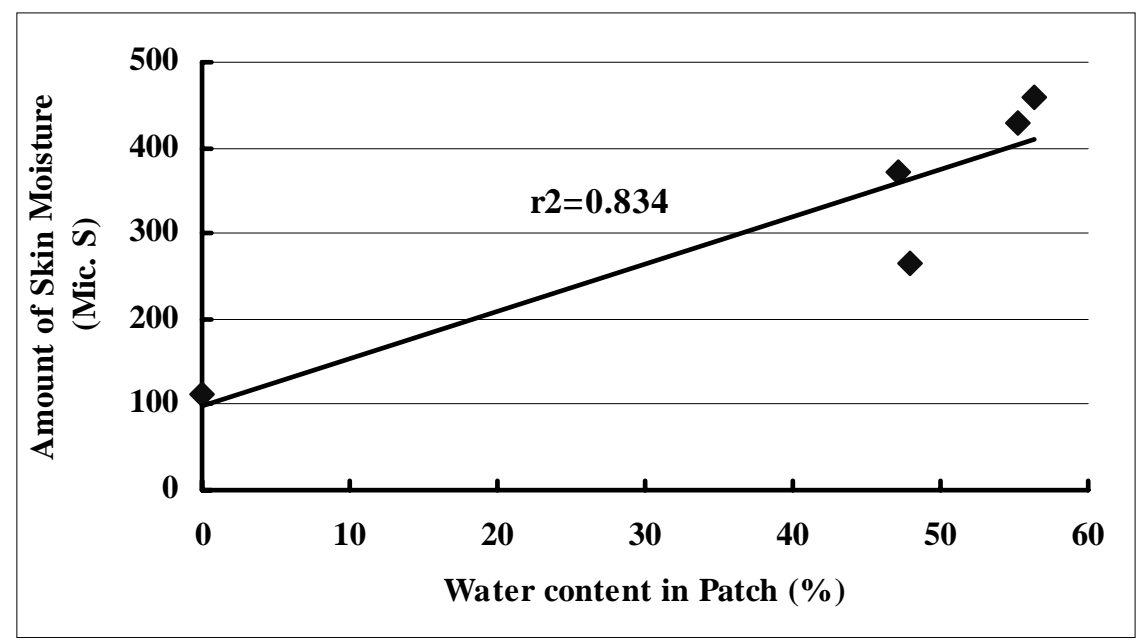

Fig. 9. Relation between water content in the patches and amount of skin moisture after patch application. (In-house data) 
stratum corneum. Peeling intensity is considered to be a parameter of pain and independent of the amount of stripped stratum corneum and skin moisture.

In conclusion, since the amount of stripped stratum corneum with the tape-type patch was here found to be higher than that with the poultice-type patch, the bio-adhesive strength of the latter was clearly lower. The amount of stripped stratum corneum and the skin moisture are correlated after removal of the tape-type patch, but not the poultice-type patch, because of its hydration effects. As for mechanical conditions, when the patch is removed, it is important to remove it as slowly as possible and horizontally, as well as to avoid a rise in skin temperature. Finally, when a patch is applied to a region of low skin moisture, the amount of stripped stratum corneum may increase accordingly.

\section{REFERENCES}

Andersen, P.H., Nangia, A., Bjerring, P. and Maibach, H.I. (1991): Chemical and pharmacologic skin irritation in man. Contact Dermatitis, 25, 283289.

Bucks, D. and Maibach, H.I. (1999): Occlusion does not uniformly enhance penetration in vivo. In Percutaneous Absorption (Bronaugh, R.L. and Maibach, H.I., eds.), vol. 97, pp.81-105, Marcel Dekker, New York.

Chien, Y.W. (1992): Transdermal drug delivery and delivery systems. In Novel Drug Delivery Systems, vol. 50, pp.301-380, Marcel Dekker, New York.

CMC. (1968): Tennenkei polymer. In Hydrophilic Polmer, vol. 102, pp.147-243, Tokyo Japan (Japanese).

Cordero, J.A., Alarcon, L., Escribano, E., Obach, R. and Domenech, J. (1997): A comparative study of the transdermal penetration of a series of nonsteroidal antiinflammatory drugs. J. Pharm. Sci., 86, 503-508.

Flannigan, S.A., Tucker, S.B., Key, M.M., Ross, C.E., Fairchild, E.J., Grimes, B.A. and Harrist, R.B. (1985): Primary irritant contact dermatitis from synthetic pyrethroid insecticide exposure. Arch. Toxicol., 56, 288-294.

Fluhr, J.W., Kuss, O., Diepgen, T., Lazzerini, S., Pelosi, A., Gloor, M. and Berardesca, E. (2001): Testing for irritation with a multifactorial approach: Comparison of eight non-invasive measuring techniques on five different irritation types. Br. J. Dermatol., 145, 696-703.
Iizuka, T., Minegishi, S., Hiramoto, K., Samizo, K., Ishizuka, H. and Ishikura, C. (1998): Relationship between skin irritation and the amount of stripped stratum corneum following the use of adhesive tape for the treatment of ischemic heart disease. Byoin Yakugaku, 24, 526-532 (in Japanese).

Inoue, K., Kuroda, K. and Nii, T. (1994): Skin stimulus of nitrate compound tapes. Shinryo to Shinyaku, 31, 203-205 (in Japanese).

Japanese Standards Association (1991): Testing methods of pressure sensitive adhesive tapes and sheets. In JIS Z 0237, pp.1-19, Japan.

Kaelble, D.H. (1959): Theory and analysis of peel adhesion: Mechanisms and mechanics. Trans. Soc. Rheol., 3, 161-180.

Kashibuchi, N. and Muramatsu, Y. (1989): Exfoliative cytology for morphological evaluation of skin. J. Soc. Cosmet. Chem. Jpn., 23, 55-57 (in Japanese).

Kawakami, J., Shinkai, N., Kawamura, N., Noguchi, K. and Kawamoto, T. (2000): In-house report.

Kawamura, N., Sugizaki, Y., Noguchi, K., Kimura, T., Okumura, Y., Saitoh, H. and Shinkai, N. (2003): In-house report.

Kay-Mugford, P., Benn, S.J., LaMarre, J. and Conlon, P. (2000): In vitro effects of nonsteroidal antiinflammatory drugs on cyclooxygenase activity in dogs. Am. J. Vet. Res., 61, 802-810.

Keiei Kaihatsu Center Syuppanbu (1981): Gelatin. In Hydrophlic Polymer Mizu-bunnsan-kata jyushino-saishinkakou kaishitu-gijyutu-to youtokaihatsu, pp.166-185, Tokyo Japan (in Japanese).

Kinoshita, T., Akemi, H. and Ohtsuka, S (1993): Preparation design and pharmaceutics basic knowledge of percutaneous absorption pharmaceuticals. Shinryo to Shinyaku, 30, 166-175 (in Japanese).

Masuda, Y. (1995): Predictivity of safety. Safety test by laboratory animals. Jpn. J. Clin. Pharmacol. Ther., 26, 545-550.

Nihon Nenchakuzai Tape Kogyokai (1995): Nenchaku-no-gainen. In Nentyaku Hand-book pp.19-70, Tokyo Japan (in Japanese).

Obata, M. and Tagami, H. (1989): Electrical determination of water content and concentration profile in a simulation model of in vivo stratum corneum. J. Invest. Dermatol., 92, 854-859.

Rosati, G.., Morisetti, A. and Tirone, P. (1992): Toxicity in animals and safety in humans: The predictive value of animal studies. Toxicol. Lett., 64/ 
Skin irritation due to patches.

65, 705-715.

Satoh, A. and Hotta, H. (1996): Changes in sensory function during aging. Jpn. J. Neuropsychopharmacol., 18, 263-267 (in Japanese).

Satoh, K. (1993): Tacky adhesion test. Setchaku, 37, 372-380 (in Japanese).

Satoh, K. (1998): Viscoelasticity and tackiness of pressure sensitive adhesives. Kobunshi Kako, 47, 371-379 (in Japanese).

Schaefer, H. and Redelmeier, T.E. (1996a): Structure and dynamics of the skin barrier. In Skin Barrier, pp.1-42, S. Karger AG, Basel.

Schaefer, H. and Redelmeier, T.E. (1996b): Composition and Structure of the Stratum corneum. In Skin Barrier, pp.43-86, S. Karger AG, Basel.

Schaefer, H. and Redelmeier, T.E. (1996c): Factors effecting percutaneous absorption. In Skin Barrier, pp.153-212, S. Karger AG, Basel.

Schaefer, H. and Redelmeier, T.E. (1996d): Factors effecting percutaneous absorption. In Skin Barrier, pp.153-212, S. Karger AG, Basel.

Shirai, S., Takiwaki, H., Kohno, H. and Arase, S. (1994): The relationship between the blood flow and the blood volume in various kinds of erythema. Rinsho Hifuka, 48, 19-23 (in Japanese).

Sugii, T., Konno, M. and Wada, S. (1989): The development of an advanced moisture permeable transparent dressing. Nitto Giho, 27, 42-47 (in Japanese).

Tagami, H., Ohi, M., Iwatsuki, K., Kanamaru, Y., Yamada, M. and Ichijo, B. (1980): Evaluation of the skin surface hydration in vivo by electrical measurement. J. Invest. Dermatol., 75, 500-507.

Tagami, H. (1981): In vivo measurement of the water content in the stratum corneum using high frequency current. Fragrance J., 46, 89-93 (in Japanese).

Tagami, H. (1982):Evaluation of moisturizing efficacy of topical agents with in vivo water sorptiondesorption test using electromeasurement. Koshokaishi, 6, 100-106 (in Japanese).

Takiwaki, H. and Serup, J. (1994): Measurement of color parameters of psoriatic plaques by narrowband reflectance spectrophotometry and tristimulus colorimetry. Skin Pharmacol., 7, 145-150.

Tomita, Y. and Tagami, H. (1993): Skin irritation study of an improved version (TY-0161) of Fandol ${ }^{\circledR}$ Tape-S: Comparison with other nitrate preparations. Kiso to Rinsho, 27, 1536-1547 (in Japa- nese).

Tukey, J.W. (1953): Some selected quick and easy methods of statistical analysis. Trans. N. Y. Acad. Sci., 16, 88-97.

Urahama, K. and Tanaka, Y. (1996): Explanation of adhesive test method. Dependence of adhesive properties on peeling conditions. Setchaku no Gijutsu, 16, 22-27 (in Japanese).

Von Zglinicki, T., Lindberg, M., Roomans, G.M. and Forslind, B.(1993): Water and ion distribution profiles in human skin. Acta. Derm. Venereol., 73, 340-343.

Warner, R.R., Myers, M.C. and Taylor, D.A. (1988): Electron probe analysis of human skin: Determination of the water concentration profile. J. Invest. Dermatol., 90, 218-224.

Warner, T.D., Giuliano, F., Vojnovic, I., Bukasa, A., Mitchell, J.A. and Vane, J.R. (1999): Nonsteroid drug selectivities for cyclo-oxygenage-1 rather than cyclo-oxygenage- 2 are associated with human gastrointestinal toxicity: A full in vitro analysis. Proc. Natl. Acad. Sci. USA, 96, 75637568.

Wester, R.C. and Maibach, H.I. (1995): Penetration enhancement by skin hydration. In Percutaneous Penetration Enhancers (Smith, E.W. and Maibach, H.I., eds.), pp. 21-34, CRC Press, Boca Raton.

Wester, R.C. and Maibach, H.I. (1999): Regional variation in percutaneous Absorption. In Percutaneous Absorption (Bronaugh, R.L. and Maibach, H.I., eds.), vol. 97, pp.107-32, Marcel Dekker, New York.

Yano, T., Nakagawa, A., Tsuji, M. and Noda, K. (1986): Skin permeability of various non-steroidal anti-inflammatory drugs in man. Life Sci., 39, 1043-1050.

Yokota, T. (1981): Nociceptors. Seimitsu Kikai, 47, 48-52 (in Japanese).

Yoshikuni, K., Tagami, H., Shirahama, S., Sano, T., Inoue, K. and Yamada, M. (1983): Measurement of the hydration state of the superficial horny layer in summer and winter. Evaluation of the several factors influencing the water content of the horny layer. Nihon Hifuka Gakkai Zasshi, 93, 491-495 (in Japanese).

Zhai, H., Poblete, N. and Maibach, H.I.(1998): Stripped skin model to predict irritation potential of topical agents in vivo in humans. Int. J. Dermatol., 37, 386-389. 